\title{
ScienceDirect
}

(2) Get Access Export

\section{Pregnancy Hypertension}

Volume 13, Supplement 1, October 2018, Page S19

\section{Expectant management of severe preeclampsia in a developing country: Maternal outcomes and perinatal survival}

Ernawati Ernawati ${ }^{a}$, Erliana Erliana ${ }^{a}$, Agus Sulistono ${ }^{a}$, Hermanto Tri Joewono a , M.I Aldika Akbar ${ }^{\text {a }}$, Budi Wicaksono ${ }^{a}$, Khanisyah Erza Gumilar ${ }^{a}$, Manggala Pasca Sarjana ${ }^{a}$, Nareswari Cininta ${ }^{a}$, Rozi Aryananda ${ }^{a}$, Risa Etika ${ }^{a}$, Windu Purnomo ${ }^{a}$, Nadhir Abdullah ${ }^{a}$, Erry Gumilar ${ }^{a}$, Gustaaf Dekker ${ }^{b}$, Aditiawarman Aditiawarman $^{\text {a }}$

田 Show more

https://doi.org/10.1016/j.preghy.2018.08.058

Get rights and content

\section{Background}

Management of severe preeclampsia which develops prior to 34 still debatable. Immediate delivery leads to high neonatal mortality and morbidity due to prematurity, conversely expectant management may potentially increase maternal morbidity and mortality.

Objective of this study was to determine maternal outcomes and perinatal survival achieved with expectant management in patients presenting with preeclampsia $<34$ weeks.

\section{Study Design}

A retrospective cohort analysis of outcome in patients with severe preeclampsia in a tertiary hospital in Indonesia during 3years: 165 patients (178 fetuses) managed expectantly were studied. Neonatal outcomes (birth weight, APGAR score, fetal growth restriction (FGR), intrauterine fetal death (IUFD), early neonatal death, and maternal complications (HELLP syndrome, pulmonary edema, eclampsia, renal insufficiency, and placental abruption) were registered.

Results 
25 patients had a preeclampsia disease onset <28weeks, 72 patients onset 28 0/7-30 $6 / 7$ weeks and 68 patients onset 31 0/7 -33 6/7weeks; 13 patients had twins. Mean days of prolongation was 9.3days (range 2-64). Overall neonatal survival was $64,6 \%$. Mean birth weight was $1323.08 \pm 383.55$ gram, $23,59 \%$ of the neonates had a birth weight $<10$ th centile. IUFD ocured in $7.9 \%$ of pregnancies, and $25.3 \%$ of the remaining babies died during early neonatal period. There were no neonatal survivors in those with a GA $<26$ weeks. At 26 to 27 6/7weeks GA, 38.5\% offspring survived. Regarding maternal outcome, $7.3 \%$ of the patients developed pulmonary edema, 5.5\% HELLP syndrome, $0.6 \%$ renal insufficiency and $0.6 \%$ placental abruption. In this patient cohort, none of the patients developed eclampsia, whereas one patient died post-partum due to stroke emboli.

\section{Conclusion}

Neonatal outcome in preterm severe preeclampsia depends mainly on GA at onset of preeclampsia and GA at delivery. Expectant management of preeclampsia presenting at less $<28$ weeks is not recommended in a developing country with limited neonatal resources.
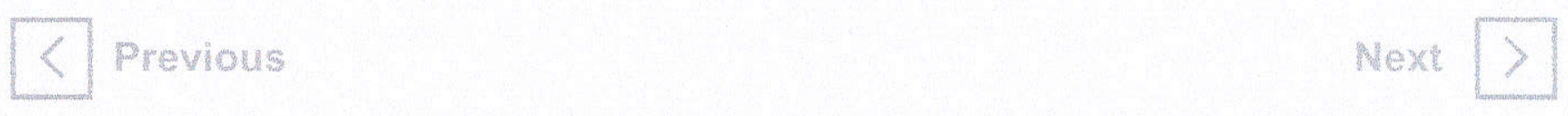

Recommended articles

ELSEVIER About ScienceDirect Remote access Shopping cart Contact and support Terms and conditions Privacy policy

We use cookies to help provide and enhance our service and tailor content and ads. By continuing you agree to the use of cookies.

Copyright (C) 2018 Elsevier B.V. or its licensors or contributors. ScienceDirect $₫$ is a registered trademark of Elsevier B.V. 
53. Expectant management of severe preeclampsia in a developing country: Maternal outcomes and perinatal survival Ernawati Ernawati ${ }^{a}$, Erliana Erliana ${ }^{a}$, Agus Sulistono ${ }^{a}$, Hermanto Tri Joewono ${ }^{a}$, M.I Aldika Akbar ${ }^{a}$, Budi Wicaksono ${ }^{a}$, Khanisyah Erza Gumilar ${ }^{a}$, Manggala Pasca Sarjana ${ }^{a}$, Nareswari Cininta ${ }^{a}$, Rozi Aryananda ${ }^{a}$, Risa Etika ${ }^{a}$, Windu Purnomo ${ }^{a}$, Nadhir Abdullah $^{a}$, Erry Gumilar ${ }^{a}$, Gustaaf Dekker ${ }^{b}$, Aditiawarman

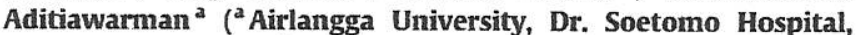
Surabaya, Indonesia, 'bdelaide University, Adelaide, Australia)

Background: Management of severe preeclampsia which develops prior to 34 still debatable. Immediate delivery leads to high neonatal mortality and morbidity due to prematurity, conversely expectant management may potentially increase maternal morbidity and mortality. Objective of this study was to determine maternal outcomes and perinatal survival achieved with expectant management in patients presenting with preeclampsia <34 weeks. Study Design: A retrospective cohort analysis of outcome in patients with severe preeclampsia in a tertiary hospital in Indonesia during 3 years: 165 patients (178 fetuses) managed expectantly were studied. Neonatal outcomes (birth weight, APGAR score, fetal growth restriction (FGR), intrauterine fetal death (IUFD), early neonatal death, and maternal complications (HELLP syndrome, pulmonary edema, eclampsia, renal insufficiency, and placental abruption) were registered.

Results: 25 patients had a preeclampsia disease onset $<28$ weeks, 72 patients onset $28 \quad 0 / 7-30 \quad 6 / 7$ weeks and 68 patients onset $310 / 7-336 / 7$ weeks; 13 patients had twins. Mean days of prolongation was 9.3 days (range 2-64). Overall neonatal survival was $64,6 \%$. Mean birth weight was $1323.08 \pm 383.55$ gram, $23,59 \%$ of the neonates had a birth weight $<10$ th centile. IUFD ocured in $7.9 \%$ of pregnancies, and $25.3 \%$ of the remaining babies died during early neonatal period. There were no neonatal survivors in those with a GA $<26$ weeks. At 26 to $276 / 7$ weeks GA, 38.5\% offspring survived. Regarding maternal outcome, $7.3 \%$ of the patients developed pulmonary edema, 5.5\% HELLP syndrome, $0.6 \%$ renal insufficiency and $0.6 \%$ placental abruption. In this patient cohort, none of the patients developed eclampsia, whereas one patient died post-partum due to stroke emboli.

Conclusion: Neonatal outcome in preterm severe preeclampsia depends mainly on GA at onset of preeclampsia and GA at delivery. Expectant management of preeclampsia presenting at less $<28$ weeks is not recommended in a developing country with limited neonatal resources.

doi:10.1016/j.preghy.2018.08.058

65. Maternal and neonatal outcomes in pregnancies complicated by Marfan syndrome a multi centre retrospective study Matthew Cauldwel ${ }^{a}$, Philip Steer ${ }^{a}$, Stephanie Curtis ${ }^{b}$, Aarthi Mohan $^{b}$, Lucy Mackillop ${ }^{c}$, Samuel Dockeree ${ }^{c}$ Suzanne Wallace $^{d}$, Gemma Malin ${ }^{d}$, Aidan Bolger ${ }^{e}$, Farah Siddiqui ${ }^{\mathrm{c}}$, Nikki Walker $^{\mathrm{f}}$, Margaret Simpson ${ }^{\mathrm{f}}$, Gemma Patridge ${ }^{\mathrm{g}}$, Dirk Wilson ${ }^{\mathrm{h}}$, James Oliver ${ }^{i}$, Helen Parry ${ }^{i}$, Emma Bredkai ${ }^{j}$, Fiona Walker ${ }^{k}$, Mark Johnson ${ }^{a}$ ( ${ }^{a}$ Imperial College London, London, United Kingdom, bSt Michaels Hospital Bristol, Bristol, United Kingdom, ' Department of Obstetrics, Oxford, United Kingdom, 'Nottingham City Hospital, Nottingham, United Kingdom, ${ }^{\mathbf{c}}$ Leicester Royal Infirmary, Leicester, United Kingdom, ${ }^{\mathbf{P}}$ Golden Jubilee Hospital Glasgow, Glasgow, United Kingdom, ${ }^{\mathrm{g}}$ Norfolk and Norwich Hospitals, Norfolk, United Kingdom, ' University Hospital of Wales, Cardiff, United Kingdom, 'iLeeds Teaching Hospitals, Leeds, United Kingdom, 'j UCLH, London, United Kingdom, ' Barts Heart Centre, London, United Kingdom)
Background: Pregnancies in Marfan's syndrome(MFS) have higher rates of maternal and neonatal complications. However, there are few large studies to evaluate these complications.

Method: Retrospective cohort study of women with MFS managed between 1st January 1998 and 1st March 2018, from 11 UK centres providing care for women with cardiac disease. Data on livebirths, miscarriages, terminations and maternal cardiac complication was collated. Complications were defined as: aortic dissection, need for cardiac intervention antepartum or up to 6 weeks postpartum and deterioration in left systolic function. Neonatal complications included delivery at $<37$ weeks and small for gestational age (SGA, birthweight $<10$ th centile). We examined aortic size prior to pregnancy and at least 6 weeks postpartum.

Results: There were 212 pregnancies in 133 women, resulting in 186 livebirths, 17 miscarriages and 9 terminations. Median age at first pregnancy $(n=130)$ was 28.5 years (IQR $23.8-33.0$ ). Only $58 \%$ in their 1st pregnancy in whom data was available had documented preconception counselling prior to pregnancy. Excluding three twin pregnancies, median gestation at delivery was 39 weeks, 24 (13.2\%) were preterm. The median birthweight centile was 40 ( $\mathrm{n}=$ 153, IQR 16.5-68.5) and 22 (12.1\%) deliveries were SGA. Larger aortic root measurements pre-pregnancy were strongly associated with earlier delivery (excluding one case with root diameter $=81 \mathrm{~mm}, \mathrm{n}$ $=86, \mathrm{p}=0.002$ ) . In $41 \%$ pregnancies women took beta-blockers Beta blocker use was associated with slightly smaller babies but this was not significant. Four women suffered aortic dissections (one Type A, four Type B all were in the puerperium), four others required root replacement within six weeks of delivery and one at 23 weeks. Three developed left systolic dysfunction during pregnancy. Five women had an increase in aortic diameter $>3 \mathrm{~mm}$ during pregnancy, but overall aortic diameters did not change significantly.

Conclusions: Pregnancy in MFS is high risk for the mother, with favourable neonatal outcomes, yet pre-pregnancy counselling is not universal.

doi:10.1016/j.preghy.2018.08.059

67. Birthweight in pregnancies complicated by maternal heart disease

Matthew Cauldwel ${ }^{1}$, Philip Steer ${ }^{1}$, Aarthi Mohan ${ }^{2}$, Adam Jakes ${ }^{3}$, Cathy Head ${ }^{3}$, Monique Sterrenberg ${ }^{4}$, Suzanne Wallace ${ }^{5}$, Gemma Malin $^{5}$, Thomas Everett ${ }^{6}$, Gemma Ulivi ${ }^{6}$, Margaret Simpson ${ }^{7}$, Mark Johnson ${ }^{1}$ ( ${ }^{1}$ Imperial College London, London, United Kingdom, ${ }^{2}$ St Michaels Hospital Bristol, Bristol, United Kingdom, ${ }^{3}$ Guys and St Thomas' Hospital, Guys and St Thomas' Hospital, London, United Kingdom, ${ }^{4}$ Queen Annes Hospital, Southampton, United Kingdom, ${ }^{5}$ Nottingham City Hospital, Nottingham, United Kingdom, ${ }^{6}$ Leeds Teaching Hospitals, Leeds, United Kingdom, ${ }^{7}$ Golden Jubilee Hospital Glasgow, Glasgow, United Kingdom)

Objective: To assess the mean and centile birthweight distribution in women with various groups of heart disease compared with controls.

Methods: Data on birthweight and gestational age at birth $(\geqslant 24$ weeks gestation) were collected about women with known heart disease (both congenital and acquired) from seven specialist UK maternity units. Women were assigned to one of 16 groups according to their cardiac lesion. Whenever possible, data on two controls delivering before and after the index cases was also collected. Birth weight percentiles (corrected for gestational age, sex and parity) were calculated using the Aberdeen norms. Using multivariate regression, we also assessed the impact of beta blockers, maternal 